\title{
EDITORIAL
}

\section{Home mechanical ventilation: who cares about how patients die?}

\author{
W. Windisch
}

$\mathrm{H}$ ome mechanical ventilation (HMV) is increasingly being used to treat chronic hypercapnic respiratory failure arising from different aetiologies, such as restrictive thoracic disorders, neuromuscular disorders (NMD), obesity hypoventilation syndrome and chronic obstructive pulmonary disease (COPD) [1-3]. The prognosis of patients receiving HMV depends on both the type and state of disease upon commencement of HMV, with the worst outcome reported in patients with COPD, as well as those with rapidly progressive NMD such as amyotrophic lateral sclerosis (ALS) [3-6]. However, in contrast, the survival benefits provided by HMV in individuals with restrictive thoracic disorders or stable, slowly progressive NMD are overwhelming [3-6]. Importantly, HMV has also been shown to strongly improve health-related quality of life (HRQoL) in patients with varying disorders including COPD and NMD [7]; this improvement was revealed via the application of the Severe Respiratory Insufficiency Questionnaire [8, 9], an instrument specifically developed and validated for patients with HMV use. In addition, enhancements in HRQoL were also reported in several previous trials that used various instruments to assess HRQoL [3-5]. Thus, HMV has become the treatment of choice for patients with chronic hypercapnic respiratory failure, which is a sign of major progress in medicine.

Nevertheless, the clinical status of all these patients will eventually deteriorate, ultimately leading to death. This scenario is characterised by the more frequent occurrence of threatening symptoms, most likely dyspnoea, and possibly problems related to ventilation, such as more alarm announcements or a greater dependence on daily ventilation. This can occur in the weeks or even months prior to death. Another important factor is the role of family members, who are often involved in the daily management of HMV patients; this is undoubtedly a huge challenge. Of note, spending time with family and friends was identified as the highest amongst 26 end-of-life priorities for different groups of terminally ill patients including those with COPD, directly followed by the wish of maintaining control over symptoms (pain and breathing comfort) [10]. Finally, family members, and even general practitioners, are possibly overburdened by the decision about when to withhold or withdraw life-sustaining therapy and how to manage symptoms in the terminal phase, particularly when information about prognosis is lacking. Therefore, the

CORRESPONDENCE: W. Windisch, Dept of Pneumology, University Hospital Freiburg, Killianstrasse 5, D-79106, Freiburg, Germany. E-mail: wolfram.windisch@uniklinik-freiburg.de decision about whether to admit terminally ill, clinically deteriorating HMV patients to hospital is accompanied by a sense of uncertainty and peradventure and, for this reason, it is essential that terminal HMV patients are also involved in the end-of-life decision making process.

In the past, decisions about end-of life issues have been investigated in hospitalised terminally ill patients. For example, a large prospective international trial on end-of-life practices in 37 intensive care units (ICUs) revealed that the practice of limiting life-sustaining treatment in European ICUs is common, but variable [11]. Very recently, structural variables such as the nurse-per-bed-ratio or the presence of an ICU specialist have been identified to be associated with the substantial variation in the incidence and characteristics of decisions to forgo life-sustaining therapies [12]. In addition, the Task Force of the European Respiratory Society on ethics and decision-making in end-stage lung disease revealed that an end-of-life decision is reached in $21.5 \%$ of patients in European respiratory ICUs where the withholding of treatment, do-notintubate/do-not-resuscitate orders and noninvasive ventilation (NIV) as the ventilatory care ceiling account for the most common procedures [13].

In contrast, there is no conclusive information about end-oflife issues and decision-making in seriously ill patients with HMV. Furthermore, there is a clear lack of information about the specific circumstances surrounding the last weeks and months of the HMV patient's life within the home environment. Physicians caring for HMV patients typically meet the patient in hospital, rather than at home; thus, we are unable to gauge the kind of needs that both patients and their carers might have in the terminal phase. This obviously refers not only to end-of-life decision making, but also directly to the challenges of managing changes in ventilation procedures in the home.

In this issue of the European Respiratory Journal, VITACCA et al. [14] present an Italian multicentre trial on the family's perception of HMV patient care during the last 3 months of life. Six domains of end-of-life care were addressed by a questionnaire which was developed for the purpose of the study and submitted to relatives of 168 deceased patients $(65 \%$ with additional long-term oxygen therapy), these were: symptoms, awareness of disease, family burden, dying, medical problems and technical problems. $69(41 \%)$ patients had COPD and $42(25 \%)$ patients had ALS, while $57(34 \%)$ patients had various underlying disorders. Remarkably, 92 $(55 \%)$ patients received invasive ventilation via tracheostoma, 
and $76(45 \%)$ patients received NIV. No dedicated or structured palliative home-care service was available during the study.

The response rate was $98.8 \%$ (168 out of 170 relatives); this is quite remarkable and obviously reflects the desire of relatives to share their experiences after their loved ones have deceased. According to the relatives' responses, $81 \%$ of patients had respiratory symptoms, with NIV patients being more likely to suffer from respiratory symptoms than those with invasive ventilation (OR 2.94, $\mathrm{p}=0.024$ ). Sedatives and opiates were only administered to $16 \%$ of patients, and drug adjustments were made by specialists (pulmonologists) in only $36 \%$ of the cases. Managing dyspnoea and other respiratory symptoms is a major goal in end-of-life care, and this goal was obviously not achieved in the majority of patients. Most patients seemed to be aware of the severity and prognosis of the disease, with $84 \%$ of patients being aware of their worsening disease state thus reflecting sufficient patientphysician communication about end-of-life. This observation, however, contrasts with a previous finding in oxygendependent COPD patients, where important areas of communication about how long patients might have to live and what dying might be like were poorly addressed [15]. The burden on the patient's family was reportedly high, since families received only minimal help from the national healthcare system or other services, while the financial burden became excessively high in $17 \%$ of the cases.

Interestingly, 90 (54\%) patients were hospitalised during the clinical deterioration phase before death, with death occurring in hospital, often in the ICU. The authors speculated that this event could have been considered as a "failure" of the end-oflife care protocol, but they also correctly refer to the difficulties in predicting non-cancer deaths. Death during HMV occurred in $55 \%$ of patients, but this was significantly less likely in the case of NIV compared with invasive ventilation (OR 0.26, $\mathrm{p}<0.001$ ). In all patients (ALS patients), the emergency number was called in $27 \%(30 \%)$ of cases and resuscitation manoeuvres were performed in $27 \%(14 \%)$ of cases. The use of healthcare resources in the last 3 months before death was reported to be very high. Finally, although there was some need for changing the ventilator settings or modifying the time spent under mechanical ventilation, technical problems were rare.

The study by VITACCA et al. [14] does, however, have some limitations, which have also been acknowledged by the authors. First, the study was retrospectively performed, and we do not know if the findings are an accurate reflection of the final months of life. Secondly, the questionnaire, which served as the primary tool for data assessment, has never been tested for its psychometric properties; most importantly, reliability and validity. Thirdly, the study was only performed in Italy and the impact of religious affiliation was not addressed; spiritual support was only provided for $20 \%$ of patients. However, a significant impact of religious affiliations and cultures on various end-of-life decisions has been noted, at least in the ICU population [16]. For this reason, generalisation of the findings appears to be hindered. Finally, only relatives and not patients were interviewed. While the importance of assessing the relatives' attitude towards end-of-life issues is undisputed, the study focuses on issues relevant for both the relatives (for example: domain "family burden") and the patients (for example: domain "control of symptoms"). Therefore, the latter cannot be reliably assessed without directly interviewing the patients concerned.

Nevertheless, despite its limitations, the study by VITACCA et al. [14] is extremely relevant and teaches us that research in the field of HMV should not only focus on physiological, epidemiological, technical, structural, financial and outcome aspects, but should also target the question of how patients with HMV die and how we can improve HRQoL in the terminal phase so as to enhance the quality of dying. According to the present study, areas for potential improvement need to incorporate symptom control, family burden, hospital admissions and the process of dying itself.

Who are the people who care most about how HMV patients die? Obviously, family members do, but both patients and their relatives need our help. To enable this intention to be fulfilled more information and, hence, more research is needed to characterise the specific circumstances in which HMV patients die. The work by VITACCA et al. [14] is the first study to primarily focus on end-of-life in HMV patients, and their work will hopefully stimulate the international research community to perform further studies on this highly relevant topic.

\section{STATEMENT OF INTEREST}

A statement of interest for W. Windisch can be found at www.erj ersjournals.com $/ \mathrm{misc} /$ statements.dtl

\section{REFERENCES}

1 Lloyd-Owen SJ, Donaldson GC, Ambrosino N, et al. Patterns of home mechanical ventilation use in Europe: results from the Eurovent survey. Eur Respir J 2005; 25: 1025-1031.

2 Janssens JP, Derivaz S, Breitenstein E, et al. Changing patterns in long-term noninvasive ventilation: a 7-year prospective study in the Geneva Lake area. Chest 2003; 123: 67-79.

3 Mehta S, Hill NS. Noninvasive ventilation. Am J Respir Crit Care Med 2001; 163: 540-577.

4 Simonds AK. Home ventilation. Eur Respir J 2003; 22: Suppl. 47, $38 \mathrm{~s}-46 \mathrm{~s}$.

5 Ambrosino N, Carpenè N, Gherardi M. Chronic respiratory care for neuromuscular diseases in adults. Eur Respir J 2009; 34: 444-451.

6 Simonds AK, Elliott MW. Outcome of domiciliary nasal intermittent positive pressure ventilation in restrictive and obstructive disorders. Thorax 1995; 50: 604-609.

7 Windisch W. Impact of home mechanical ventilation on healthrelated quality of life. Eur Respir J 2008; 32: 1328-1236.

8 Windisch W, Freidel K, Schucher B, et al. The Severe Respiratory Insufficiency (SRI) Questionnaire: a specific measure of healthrelated quality of life in patients receiving home mechanical ventilation. J Clin Epidemiol 2003; 56: 752-759.

9 Windisch W, Budweiser S, Heinemann F, et al. The Severe Respiratory Insufficiency (SRI) Questionnaire was valid for patients with COPD. J Clin Epidemiol 2008; 61: 848-853.

10 Downey L, Engelberg RA, Curtis JR, et al. Shared priorities for the end-of-life period. J Pain Symptom Manage 2009; 37: 175-188.

11 Sprung CL, Cohen SL, Sjokvist P, et al. End-of-life practices in European intensive care units: the Ethicus Study. JAMA 2003; 290: 790-797.

12 Azoulay E, Metnitz B, Sprung CL, et al. End-of-life practices in 282 intensive care units: data from the SAPS 3 database. Intensive Care Med 2009; 35: 623-630. 
13 Nava S, Sturani C, Hartl S, et al. End-of-life decision-making in respiratory intermediate care units: a European survey. Eur Respir J 2007; 30: 156-164

14 Vitacca M, Grassi M, Barbano L, et al. Last 3 months of life in home-ventilated patients: the family perception. Eur Respir J 2010, 35: 1064-1071.
15 Curtis JR, Engelberg RA, Nielsen EL, et al. Patient-physician communication about end-of-life care for patients with severe COPD. Eur Respir J 2004; 24: 200-205.

16 Sprung $\mathrm{CL}$, Maia $\mathrm{P}$, Bulow $\mathrm{HH}$, et al. The importance of religious affiliation and culture on end-of-life decisions in European intensive care units. Intensive Care Med 2007; 33: 1732-1739. 